\title{
FUSE observations of hot white dwarfs
}

\author{
B. Wolff ${ }^{1}$, J. W. Kruk 2 , D. Koester ${ }^{1}$, N. F. Allard ${ }^{3,4}$, R. Ferlet ${ }^{3}$, and A. Vidal-Madjar ${ }^{3}$ \\ 1 Institut für Theoretische Physik und Astrophysik, Universität Kiel, 24098 Kiel, Germany \\ e-mail: wolff, koester@astrophysik.uni-kiel.de \\ 2 Center for Astrophysical Sciences, The Johns Hopkins University, Baltimore, MD, 21218, USA \\ e-mail: kruk@pha.jhu.edu \\ 3 CNRS Institut d'Astrophysique de Paris, 98bis boulevard Arago, 75014 Paris, France \\ e-mail: allard, ferlet, alfred@iap.fr \\ 4 Observatoire de Paris-Meudon, Département Atomes et Molécules en Astrophysique, \\ 92195 Meudon Principal Cedex, France
}

Received 6 April 2001 / Accepted 26 April 2001

\begin{abstract}
We have analyzed FUSE observations of six hot white dwarf stars: four DA white dwarfs with $T_{\text {eff }} \gtrsim$ $45000 \mathrm{~K}$, the DAO Feige $55\left(T_{\text {eff }} \approx 55000 \mathrm{~K}\right)$, and the DA CD $-38^{\circ} 10980\left(T_{\text {eff }} \approx 24000 \mathrm{~K}\right)$. Photospheric lines from SiIV, P V, and S VI can be observed in the majority of the five hotter objects. Feige 55 shows also several other heavier elements. The measured abundances agree only partly with the predictions of the radiative levitation theory. We attribute this to current limitations of the models and the probable presence of mass loss. In the spectrum of $\mathrm{CD}-38^{\circ} 10980$, we have observed the quasi-molecular satellites of $\mathrm{L} \beta$. This confirms theoretical predictions about the visibility range for these features.
\end{abstract}

Key words. stars: abundances - stars: atmospheres - white dwarfs - ultraviolet: stars

\section{Introduction}

The ultraviolet part of the electromagnetic spectrum is of special interest for investigations of hot white dwarf stars because of strong lines from hydrogen, helium, and several heavier elements. Our knowledge of the chemical composition of white dwarf atmospheres depends strongly on observations in this spectral region. However, the ultraviolet is still not explored fully. At wavelengths longer than $\mathrm{L} \alpha$, intensive studies could be realized mainly with the International Ultraviolet Explorer (IUE) and the Hubble Space Telescope (HST). At shorter wavelengths, only very few observations of white dwarfs obtained with the Voyager spectrometers, the Hopkins Ultraviolet Telescope (HUT), and the Orbiting and Retrievable Far and Extreme Ultraviolet Spectrometer (ORFEUS) exist until now.

The Far Ultraviolet Spectroscopic Explorer (FUSE) provides for the first time the possibility to investigate the far ultraviolet at wavelengths shorter than $\mathrm{L} \alpha$ for a significant number of white dwarfs. FUSE is a NASA astronomy satellite aiming at high resolution spectroscopy from 905 to $1187 \AA$. It has been developed in cooperation with the Canadian Space Agency and the Centre National d'Etudes Spatiales of France. An overview of the FUSE mission has been given by Moos et al. (2000).

Send offprint requests to: B. Wolff,

e-mail: wolff@astrophysik. uni-kiel.de
In this paper, we analyze FUSE spectra of six white dwarfs with hydrogen-rich atmospheres: four DAs with $T_{\text {eff }} \gtrsim 45000 \mathrm{~K}$, the DAO Feige $55\left(T_{\text {eff }} \approx 55000 \mathrm{~K}\right)$, and the cooler DA CD $-38^{\circ} 10980\left(T_{\mathrm{eff}} \approx 24000 \mathrm{~K}\right)$. These objects were observed within a larger project investigating the local interstellar medium. We concentrate here on the photospheric lines to gain an improved knowledge of the atmospheric composition of the five hotter white dwarfs. CD $-38^{\circ} 10980$ is interesting because of the possibility to study the $\mathrm{L} \beta$ satellite features.

\section{Observations and data reduction}

The observations used in this work are listed in Table 1. WD 2309+105 (GD 246) and WD 2331-475 were observed through the LWRS aperture (30" square); the other stars were all observed through the MDRS aperture $\left(4^{\prime \prime} \times 20^{\prime \prime}\right.$ slit). The channel alignment is satisfactory for the LWRS observations, with stable fluxes being obtained in all channels (see Sahnow et al. 2000 for a discussion of the alignment issues for the four FUSE telescopes). The MDRS observations, however, are affected by channel misalignments. These observations were generally obtained fairly early in the mission, before the channel alignment behavior was well-characterized and while MDRS observing procedures were still being developed. As a consequence, the effective exposure time for the $\mathrm{LiF} 2, \mathrm{SiC} 1$, and $\mathrm{SiC} 2$ channels can be lower than that shown in Table 1 . The LiF1 
Table 1. Log of observations.

\begin{tabular}{lllr}
\hline WD Number & Data set & Date & Exp./s \\
\hline $0455-282$ & P1041101 & $2000-02-03$ & 19668 \\
& P1041102 & $2000-02-04$ & 10121 \\
& P1041103 & $2000-02-07$ & 17677 \\
$1202+608$ & P1042101 & $2000-02-26$ & 13763 \\
& P1042105 & $1999-12-29$ & 19638 \\
$1620-391$ & Q1100101 & $2000-07-18$ & 4830 \\
$1631+781$ & P1042901 & $2000-01-18$ & 22300 \\
& P1042902 & $2001-01-31$ & 30160 \\
$2309+105$ & P1044101 & $2000-07-19$ & 14828 \\
$2331-475$ & P1044201 & $2000-06-23$ & 19356 \\
\hline
\end{tabular}

channel is essentially always aligned, with flux variations between exposures usually being less than $1 \%$. The LiF2 channel alignment is fairly good in most cases, providing $50 \%$ to $100 \%$ of the exposure time (except that detector 2 was off for P1041101). The alignment of the $\mathrm{SiC}$ channels is more variable. The effective exposure time for the $\mathrm{SiC}$ channels ranges from zero (for WD 0455-282 and the first observation of WD 1631+781) to nearly $100 \%$.

The raw data were processed using the standard calibration pipeline (CALFUSE version 1.8.7), which produces a calibrated spectrum for each exposure in each channel. The relative alignment of the channels would often change from one exposure to the next, causing the star to be placed in different locations in the spectrograph entrance aperture. The resulting shifts in the wavelength scale were negligible for the MDRS observations. For the LWRS observations, the processed spectra for each exposure were shifted manually as needed to correct for this effect. In addition, the WD $1202+608$ (Feige 55) spectra were shifted to coalign the photospheric absorption lines in order to correct for the orbital motion of this binary system. The spectra for each channel were averaged following coalignment of the individual exposures.

In most cases an absorption line would appear in two to four channels; comparison of the spectra from different channels thus served to verify that none of the lines being fit were affected by instrumental artifacts. In order to increase the signal-to-noise ratio for interesting parts of the spectra, the observations for each channel were resampled onto a common wavelength scale (with $0.006 \AA$ resolution) and combined. If the effective exposure times varied significantly then the individual spectra were weighted with the apparent flux level.

\section{Photospheric composition}

\subsection{Hot DA white dwarfs}

\subsubsection{Overview}

The chemical composition of white dwarf atmospheres is determined mainly by the high surface gravity which leads to a chemical stratification where the lightest element present, either hydrogen or helium, floats on top of the atmosphere. For selective ions of heavier elements, the effect of gravity can be compensated if radiative acceleration is strong enough to prevent these ions from sinking downwards. Theoretical calculations and observations in the ultraviolet, extreme ultraviolet, and X-ray spectral regions have shown that radiative levitation can support ions from elements like carbon, nitrogen, oxygen, silicon, iron, nickel, and others in the atmospheres of DA white dwarfs at temperatures higher than $T_{\text {eff }} \approx 40000-50000 \mathrm{~K}$ (see e.g. Barstow et al. 1993, 1997a; Wolff et al. 1998; and references therein).

The four hot DA white dwarfs of our sample are with $T_{\text {eff }} \gtrsim 45000 \mathrm{~K}$ - in an interesting temperature region for the study of radiative levitation. We have analyzed the FUSE spectra of GD 246 (WD 2309+105), MCT 0455-2812 (WD 0455-282, RE J0457-28), MCT 2331-4731 (WD 2331-475, RE J2334-47), and RE J1629+78 (WD 1631+781). The most prominent features in all spectra are the broad photospheric Lyman lines from $\mathrm{L} \beta$ to the series limit. In addition, sharp interstellar hydrogen features are always visible in the line cores. Further interstellar lines arise from several ground states of neutral or low ionized heavier elements. Their Doppler velocities are compatible with the hydrogen cores. The strongest interstellar metal features are the N I lines near $1134 \AA$, C II at $1036.34 \AA$, O I at 1039.23 Si II at $989.87 \AA$, and Fe II at $1144.94 \AA$. A complete list can be found in Table 2 .

The spectra of MCT 0455-2812, MCT 2331-4731, and GD 246 show a second system of metal lines comprising several higher ionized species (Si IV, P IV, P V, S IV, SVI) which are most probably of photospheric origin. Their velocities are generally well-separated from the velocities of the interstellar lines. Very prominent are the P V doublet at 1117.98/1128.01 $\AA$ and the SiIV doublet at $1122.49 / 1128.34 \AA$. Sulfur could be detected in three objects for the first time. Silicon lines have already been observed in IUE spectra of GD 246 (Holberg et al. 1998). The ORFEUS observations of MCT 0455-2812 showed phosphorus and silicon (Vennes et al. 1996). Si IV, P V, and S VI are typical ions which are also detected in FUSE spectra of other hot DA white dwarfs (Chayer et al. 2000; Barstow et al. 2001). The photospheric features found in our sample are listed in Table 3. RE J1629+78 does not show photospheric metals.

\subsubsection{Analysis}

Our main interests are the atmospheric element abundances in order to improve our knowledge about the presence of heavier elements and the radiative levitation process. For this purpose, we need to know the structure of the atmosphere which is determined by the effective temperature, the surface gravity, and the chemical composition. The composition is extremely important due to the line-blanketing and backwarming effects from millions of metal lines in the extreme ultraviolet (EUV). If this is not 
Table 2. Identified interstellar metal lines in FUSE spectra of four DA white dwarfs.

\begin{tabular}{|c|c|c|c|c|c|}
\hline Ion & $\lambda / \AA$ & 0455 & 1631 & 2309 & 2331 \\
\hline \multirow[t]{2}{*}{ C II } & 1036.34 & $\times$ & $\times$ & $x$ & $\times$ \\
\hline & 1037.02 & $\times$ & $\times$ & $x$ & $\times$ \\
\hline C III & 977.02 & $\times$ & $\times$ & $x$ & $\times$ \\
\hline \multirow[t]{11}{*}{ N I } & 952.30 & & $\times$ & & \\
\hline & 953.42 & & $x$ & $\times$ & $\times$ \\
\hline & 953.66 & & $\times$ & $\times$ & $x$ \\
\hline & 953.97 & & $\times$ & $x$ & $x$ \\
\hline & 954.10 & & $x$ & $\times$ & \\
\hline & 963.99 & & $\times$ & $\times$ & $x$ \\
\hline & 964.63 & & $x$ & $\times$ & $x$ \\
\hline & 965.04 & & $\times$ & $\times$ & \\
\hline & 1134.16 & $\times$ & $\times$ & $\times$ & $\times$ \\
\hline & 1134.42 & $x$ & $\times$ & $\times$ & $x$ \\
\hline & 1134.98 & $\times$ & $\times$ & $\times$ & $x$ \\
\hline \multirow[t]{2}{*}{ N II } & 915.61 & $x$ & $\times$ & $x$ & $x$ \\
\hline & 1083.99 & & $x$ & $\times$ & $\times$ \\
\hline \multirow[t]{12}{*}{ O I } & 919.66 & & $\times$ & $x$ & $x$ \\
\hline & 921.86 & & $x$ & $x$ & $x$ \\
\hline & 924.95 & & $\times$ & $\times$ & $x$ \\
\hline & 929.52 & & $\times$ & $x$ & $x$ \\
\hline & 936.63 & & $\times$ & $\times$ & $x$ \\
\hline & 948.69 & & $x$ & $x$ & $\times$ \\
\hline & 950.88 & & $\times$ & $x$ & $\times$ \\
\hline & 971.74 & & $x$ & $x$ & $\times$ \\
\hline & 976.45 & & $\times$ & $\times$ & $\times$ \\
\hline & 988.66 & & $x$ & $x$ & $\times$ \\
\hline & 988.77 & $\times$ & $x$ & $x$ & $x$ \\
\hline & 1039.23 & $x$ & $\times$ & $\times$ & $x$ \\
\hline \multirow[t]{2}{*}{ Si II } & 989.87 & $x$ & $x$ & $x$ & $x$ \\
\hline & 1020.70 & & $\times$ & $\times$ & $\times$ \\
\hline \multirow[t]{2}{*}{ P II } & 963.80 & & $\times$ & & \\
\hline & 1152.82 & & $\times$ & & \\
\hline \multirow[t]{2}{*}{ Ar I } & 1048.22 & & $\times$ & $x$ & $\times$ \\
\hline & 1066.66 & & $\times$ & $x$ & $\times$ \\
\hline \multirow[t]{5}{*}{ Fe II } & 1063.18 & & $\times$ & $\times$ & \\
\hline & 1096.88 & & $x$ & & $x$ \\
\hline & 1121.98 & & $x$ & & \\
\hline & 1125.45 & & $x$ & $\times$ & $\times$ \\
\hline & 1144.94 & $\times$ & $\times$ & $\times$ & $\times$ \\
\hline
\end{tabular}

taken into account the effective temperature from analyses of the Balmer lines can be overestimated by several thousand degrees (see e.g. Lanz et al. 1996; Wolff et al. 1998). The most important elements for the EUV opacity are iron and nickel. The metals observed in our FUSE spectra are of minor importance and can therefore be neglected for the atmospheric structure. However, we need information about the iron and nickel content from other sources.

The four objects in our sample have been observed with the Extreme Ultraviolet Explorer (EUVE). Wolff et al. (1998) have analyzed the EUVE data of white dwarfs using the well-studied DA G 191-B2B as reference object. They could reproduce the EUVE spectrum with the same
Table 3. Identified photospheric metal lines in FUSE spectra of three DA white dwarfs.

\begin{tabular}{|c|c|c|c|c|}
\hline Ion & $\lambda / \AA$ & $\begin{array}{c}0455 \\
W_{\lambda} / \mathrm{m} \AA\end{array}$ & $\begin{array}{c}2309 \\
W_{\lambda} / \mathrm{m} \AA\end{array}$ & $\begin{array}{c}2331 \\
W_{\lambda} / \mathrm{m} \AA\end{array}$ \\
\hline \multirow[t]{4}{*}{ Si IV } & $1066.61^{*}$ & & & \\
\hline & $1066.65^{*}$ & 71 & 30 & 63 \\
\hline & 1122.49 & 54 & 24 & 62 \\
\hline & 1128.34 & 70 & 38 & 59 \\
\hline P IV & 1030.52 & 10 & & 13 \\
\hline \multirow[t]{2}{*}{ P V } & 1117.98 & 82 & 36 & 73 \\
\hline & 1128.01 & 68 & 37 & 60 \\
\hline \multirow[t]{2}{*}{ S IV } & 1062.68 & & & 11 \\
\hline & 1073.00 & 16 & & 12 \\
\hline \multirow[t]{2}{*}{ S VI } & 933.38 & 41 & & 21 \\
\hline & 944.52 & 40 & 8 & 39 \\
\hline
\end{tabular}

* Possibly blended by interstellar Ar I (1066.66 Å).

abundances as found from HST/GHRS spectra. We use their EUV opacities for our objects to calculate model atmospheres for the FUSE analysis.

Effective temperature and gravity could be determined in principle from the Lyman lines of the FUSE spectra. However, we will defer a complete detailed analysis of the broad hydrogen lines until knowledge of the flux calibration and its uncertainties have been improved, and use instead the following simpler procedure. We use the results from Finley et al. (1997) for $\log g$ and an initial value of $T_{\text {eff }}$, calculate EUV-blanketed model atmospheres, and compare the model line profiles to the observed FUSE spectra. Since Finley et al. used pure hydrogen models, we have to expect that they overestimated the effective temperatures. In this case, we decrease $T_{\text {eff }}$ until a reasonable fit to the FUSE observations can be obtained. From the fit to different Lyman lines we estimate an uncertainty of $\pm 2000 \mathrm{~K}$ for this procedure which is a sufficient accuracy for the abundance analysis.

We use line-blanketed LTE model atmospheres for the analysis of the Lyman and metal lines. The program code is able to handle the absorption from millions of metal lines in the EUV. A recent description of the procedures for the calculation of theoretical atmospheres and synthetic spectra is given by Finley et al. (1997). For the abundance determination, we use a fixed atmospheric structure as described above for each object. Synthetic spectra for the FUSE region are then calculated with various abundances for the interesting elements. The best fits are determined by a visual comparison of observed and model spectra. Typical uncertainties in the abundances caused by continuum fitting and the noise level are about $25 \%$. The results are listed in Table 4 . Some 
Table 4. Photospheric abundances relative to hydrogen (number ratios) of the hot DA white dwarfs as determined from FUSE spectra. Predicted values from Chayer et al. (1995b) are shown for comparison. For RE J1629+78, the calculation of Chayer et al. with a pure hydrogen background is used. For the other stars, we list the results calculated with an atmosphere contaminated with several heavier elements.

\begin{tabular}{lrlrllllll}
\hline & \multicolumn{2}{c}{$\mathrm{Si} / \mathrm{H}$} & \multicolumn{2}{c}{$\mathrm{P} / \mathrm{H}$} & \multicolumn{2}{c}{$\mathrm{S} / \mathrm{H}$} & \multicolumn{2}{c}{$\mathrm{Fe} / \mathrm{H}$} \\
WD Number & \multicolumn{1}{c}{ observed } & predicted & observed & predicted & observed & predicted & observed & predicted \\
\hline $0455-282^{a}$ & $6.0 \times 10^{-7}$ & $2 \times 10^{-8}$ & $1.2 \times 10^{-7}$ & n.a. & $1.0 \times 10^{-7}$ & $3 \times 10^{-6}$ & $<1.0 \times 10^{-5}$ & $2 \times 10^{-6}$ \\
$0455-282^{b}$ & $1.5 \times 10^{-6}$ & $1 \times 10^{-10}$ & $2.0 \times 10^{-7}$ & n.a. & $5.0 \times 10^{-7}$ & $6 \times 10^{-6}$ & $<1.0 \times 10^{-5}$ & $5 \times 10^{-6}$ \\
$1631+781$ & $<1.0 \times 10^{-9}$ & $2 \times 10^{-7}$ & $<4.0 \times 10^{-10}$ & n.a. & $<3.0 \times 10^{-9}$ & $1 \times 10^{-6}$ & $<2.0 \times 10^{-6}$ & $1 \times 10^{-6}$ \\
$2309+105$ & $5.0 \times 10^{-8}$ & $2 \times 10^{-8}$ & $7.5 \times 10^{-9}$ & n.a. & $<3.0 \times 10^{-7}$ & $3 \times 10^{-6}$ & $<2 \times 10^{-5}$ & $1 \times 10^{-6}$ \\
$2331-475$ & $6.0 \times 10^{-7}$ & $6 \times 10^{-9}$ & $6.0 \times 10^{-8}$ & n.a. & $3.0 \times 10^{-7}$ & $2 \times 10^{-6}$ & $<5 \times 10^{-6}$ & $5 \times 10^{-7}$ \\
\hline
\end{tabular}

${ }^{a} T_{\text {eff }}=56000 \mathrm{~K} ;{ }^{b} T_{\text {eff }}=66000 \mathrm{~K}$.

example fits are presented in Figs. 1 and 2. The resulting effective temperatures are discussed in the next section.

\subsubsection{Comments on individual objects}

For GD 246, Finley et al. (1997) have determined $T_{\text {eff }}=$ $58700 \mathrm{~K}$ and $\log g=7.8$. These values are compatible with the FUSE Lyman lines if a pure hydrogen atmosphere is used. With an atmosphere containing $25 \%$ of the G191-B2B abundances for the EUV absorption - as implied by the EUVE analysis - the Lyman series can be reproduced better with $T_{\text {eff }}=54000 \mathrm{~K}$. We use this result for the abundances listed in Table 4. However, the values do not change if a pure hydrogen atmosphere with $T_{\text {eff }}=59000 \mathrm{~K}$ is used instead. The photospheric lines in GD 246 are at $-6 \mathrm{~km} \mathrm{~s}^{-1}$ with respect to the interstellar lines, the only case for which the separation is small.

Effective temperature and gravity for MCT 2331-4731 from Finley et al. are $55800 \mathrm{~K}$ and 8.1, respectively. These values are compatible with the FUSE spectrum, but the abundance from the SVI lines is higher than the result from S IV. However, both ionization stages can be reproduced with the same value if the temperature is increased to $62000 \mathrm{~K}$. We give this abundance in Table 4 . The photospheric lines in MCT 2331-4731 are at $+27 \mathrm{~km} \mathrm{~s}^{-1}$ with respect to the interstellar lines.

The situation for MCT 0455-2812 is more difficult. Finley et al. have determined $T_{\text {eff }}=55700 \mathrm{~K}$ and $\log g=$ 7.8 whereas Barstow et al. (1997b) found from the ORFEUS Lyman spectrum $T_{\text {eff }}=66500 \mathrm{~K}$ and $\log g=$ 7.4. The EUVE spectrum could also be reproduced better with $T_{\text {eff }} \approx 66000 \mathrm{~K}$ (Dupuis et al. 1995; Barstow et al. 1997b; Wolff et al. 1998). Because we did not obtain sufficient data in the SiC channels, the FUSE data cannot be used to distinguish between both temperatures. Therefore, we list abundance determinations with both values in Table 4. As in the case of MCT 2331-4731, the S IV and S VI lines cannot be reproduced with the same abundance. The S VI value is about a factor of 10 higher even with $T_{\text {eff }}=66000 \mathrm{~K}$. We give the S IV abundance in Table 4. In MCT 0455-2812, the photospheric lines are at $+64 \mathrm{~km} \mathrm{~s}^{-1}$ with respect to the interstellar lines.

The FUSE spectrum of RE J1629+78 does not show any photospheric metal lines. This is in agreement with previous EUVE results (Wolff et al. 1999a, 1999b) indicating a pure hydrogen atmosphere. We list upper limits for $\mathrm{Si}, \mathrm{P}, \mathrm{S}$, and $\mathrm{Fe}$ determined using $T_{\text {eff }}=42500 \mathrm{~K}$ and $\log g=7.6$ (Sion et al. 1995) in Table 4 .

\subsection{The $D A O$ Feige 55}

Feige 55 (WD 1202+608) is a double-degenerate white dwarf consisting of a DAO and an unseen companion (Holberg et al. 1995b). Photospheric lines from carbon, nitrogen, oxygen, silicon, iron, and nickel could be identified in IUE spectra (Lamontagne et al. 1993; Holberg et al. 1998). The FUSE observations exhibit also numerous metal lines. The photospheric features can be easily distinguished from interstellar lines due to the orbital motion which is visible in successive exposures. The new spectra add phosphorus and sulfur to the list of identified metals in Feige 55. Interesting is the presence of the O VI lines at 1032/1038 А (see Fig. 3).

At least two interstellar components can be resolved by FUSE, separated by $45 \mathrm{~km} \mathrm{~s}^{-1}$. The blueward component has a much lower column density and appears only in the strongest lines. The velocities of the photospheric lines vary from roughly $+13 \mathrm{~km} \mathrm{~s}^{-1}$ to $+90 \mathrm{~km} \mathrm{~s}^{-1}$, relative to the stronger ISM component, during the P1042101 observation. This is consistent with the semi-amplitude of $77 \mathrm{kms}^{-1}$ and the period of 1.5 days reported by Holberg et al. (1995b).

The determination of the effective temperature from Balmer lines is problematic in Feige 55 since the higher lines require higher temperatures than the lower ones. Bergeron et al. (1993) could achieve a consistent fit to all lines by adding iron with $\mathrm{Fe} / \mathrm{H}=10^{-3}$ in addition to helium to the EUV opacity. To avoid the Balmer line 


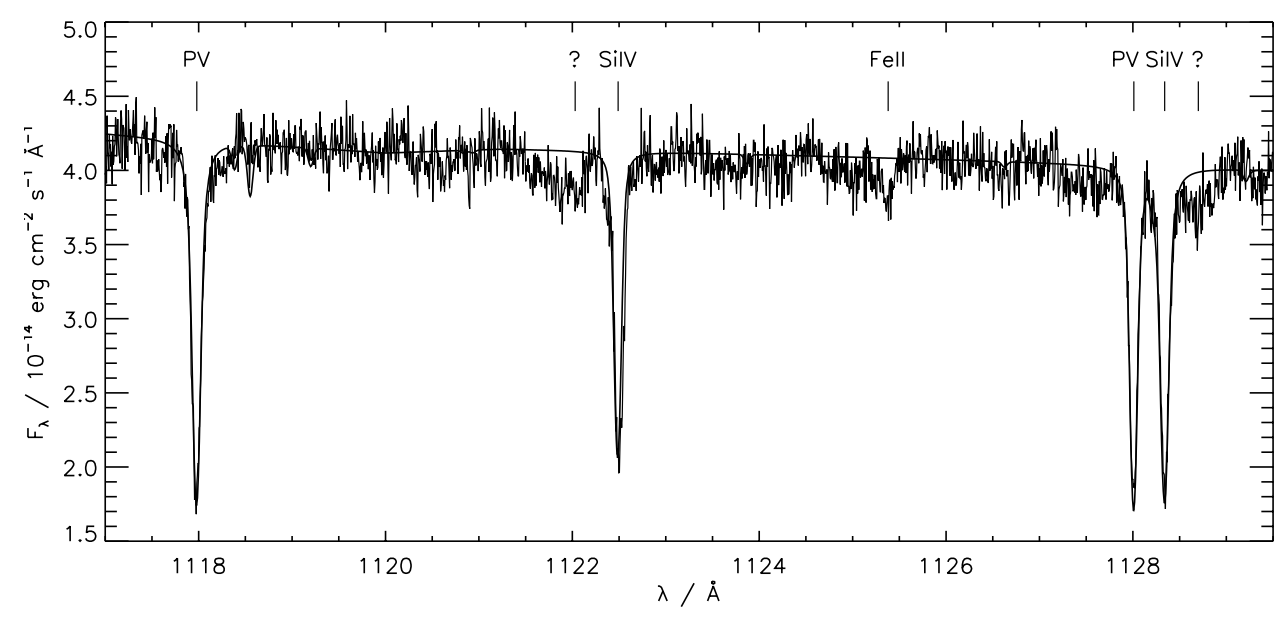

Fig. 1. Photospheric lines of Si IV and P V in the FUSE spectrum of MCT 2331-475 displayed together with a model spectrum. Also visible is an interstellar line of Fe II (1125.45 $\AA$ ). The features at $1122.03 \AA$ and $1128.70 \AA$ are possibly due to S V and S IV, respectively. They are also visible in MCT 0455-2812 and Feige 55 and take part in the orbital motion of Feige 55.

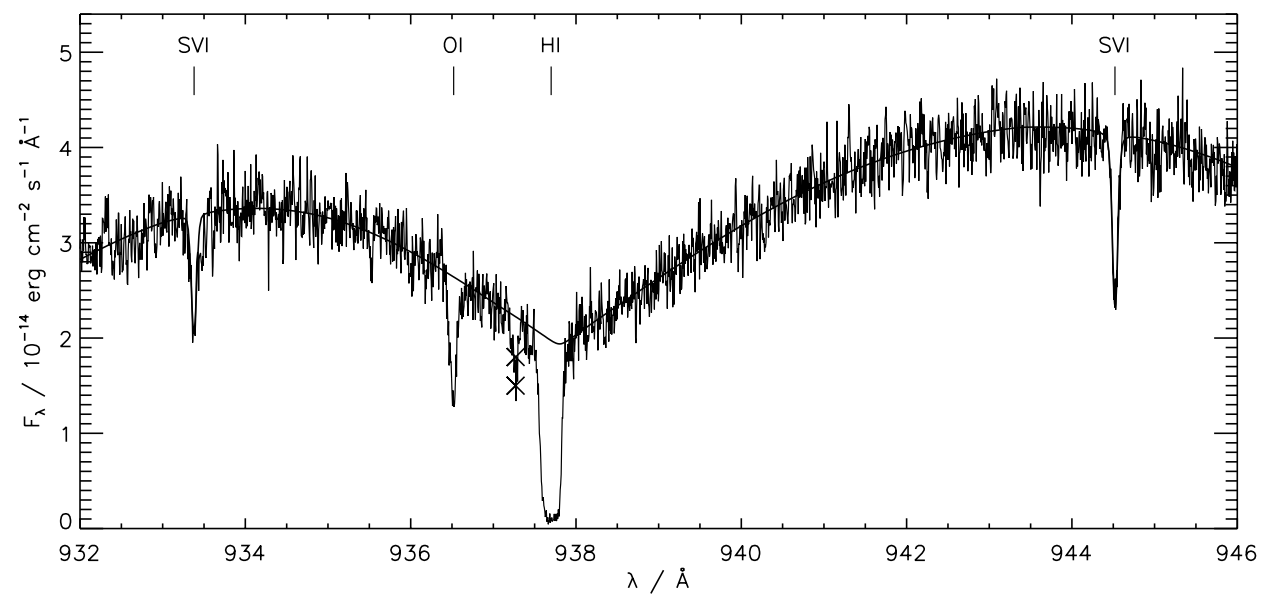

Fig. 2. Photospheric lines of S VI in the FUSE spectrum of MCT 2331-475 displayed together with a model spectrum. The feature at $937.3 \AA$ is an artifact.

problem, Bergeron et al. (1994) have used only the wings of the lines in their analysis of DAO white dwarfs. They have determined $T_{\text {eff }}=58300 \pm 1500 \mathrm{~K}, \log g=7.15 \pm 0.11$, $\log \mathrm{He} / \mathrm{H}=-2.92 \pm 0.31$, and $T_{\text {eff }}=55100 \pm 1300 \mathrm{~K}$, $\log g=6.96 \pm 0.10, \log \mathrm{He} / \mathrm{H}=-2.91 \pm 0.28$, respectively, from two optical spectra of Feige 55 .

For the following analysis, we have fixed gravity and helium abundance at the values from Bergeron et al. (1994; $\left.\log g=7.0, \mathrm{He} / \mathrm{H}=10^{-3}\right)$. We do not use the He II lines of the FUSE spectra to determine the helium abundance because these features are very broad and rather noisy and do not allow a precise analysis. The value from Bergeron et al. is, however, compatible with the FUSE lines.

$T_{\text {eff }}$ is determined with the flux distribution from the optical to the ultraviolet as given by the visual magnitude and the low resolution IUE spectrum. As a first step, we have used only hydrogen and helium as absorbers. With this result $\left(T_{\text {eff }}=65000 \mathrm{~K}\right)$, preliminary abundances for heavier elements were determined. In a second step, we added the most important EUV absorbers iron and nickel to the opacity. This gave $T_{\text {eff }}=60000 \pm 5000 \mathrm{~K}$.

The EUV opacity of helium, iron, and nickel is also taken into account for the calculation of model spectra to determine the element abundances. Two or more ionization stages are visible for most elements in the FUSE spectra. We determine the element abundances seperately from each ionization stage for three different effective temperatures. The results are shown in Table 5. In addition to the ionization stages listed in this table, there are also weak lines from $\mathrm{OV}$ and Fe VII present.

A systematic tendency can be observed in Table 5: At $T_{\text {eff }}=60000 \mathrm{~K}$, the higher ionization stage requires always higher abundances than the lower stage. The discrepancy can be removed if the temperature is increased to 70000-75000 K. These temperatures seem to be in contradiction with the overall flux distribution. However, the gradient of the flux depends only weakly on $T_{\text {eff }}$ at these temperatures (Rayleigh-Jeans region) so that errors of a few per cent in the IUE calibration or the visual magnitude could result in changes of $10000 \mathrm{~K}$. Moreover, 


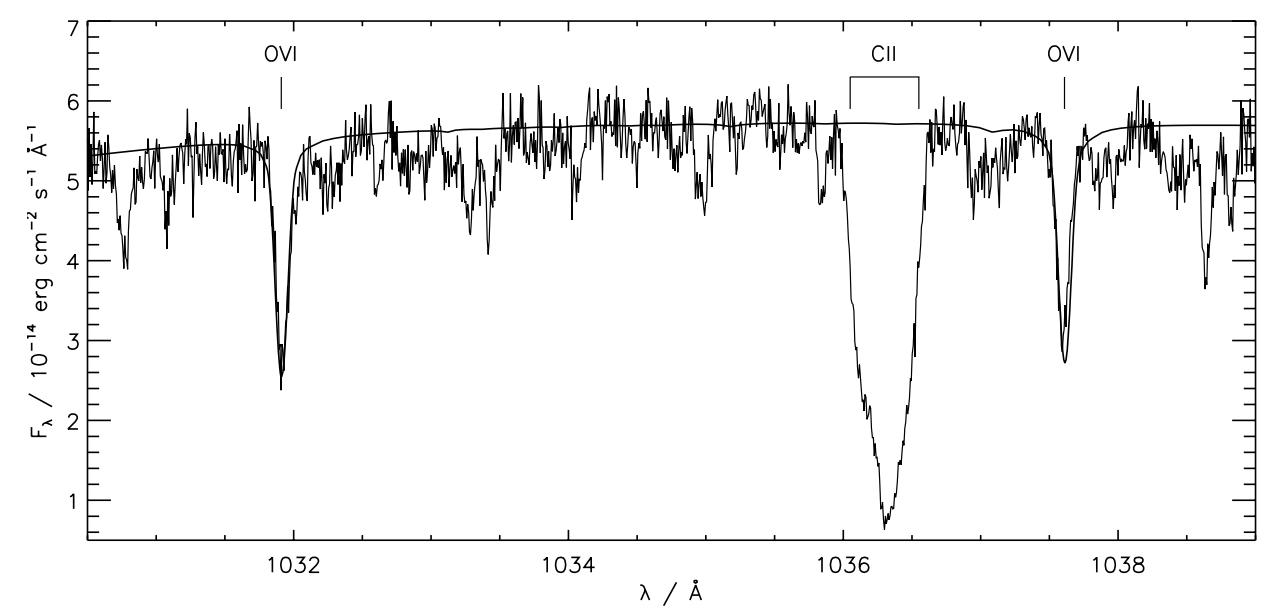

Fig. 3. O VI lines in the spectrum of Feige 55 displayed together with a model spectrum. The individual exposures were shifted according to the orbital motion and co-added. Therefore, the interstellar C II line is artificially broadened. There are also several other weak photospheric lines present.

Table 5. Photospheric abundances (number ratios relative to hydrogen) of Feige 55 for different effective temperatures as derived from different ionization stages.

\begin{tabular}{|c|c|c|c|}
\hline Ion & $T_{\text {eff }}=60000 \mathrm{~K}$ & $T_{\text {eff }}=70000 \mathrm{~K}$ & $T_{\text {eff }}=75000 \mathrm{~K}$ \\
\hline C III & $1.0 \times 10^{-6}$ & $6.0 \times 10^{-6}$ & $2.0 \times 10^{-5}$ \\
\hline C IV & $4.5 \times 10^{-6}$ & $6.0 \times 10^{-6}$ & $1.0 \times 10^{-5}$ \\
\hline N III & $1.0 \times 10^{-6}$ & $3.0 \times 10^{-6}$ & $3.0 \times 10^{-5}$ \\
\hline NIV & $2.0 \times 10^{-5}$ & $2.0 \times 10^{-5}$ & $3.0 \times 10^{-5}$ \\
\hline O III & $4.0 \times 10^{-6}$ & $1.5 \times 10^{-5}$ & $3.0 \times 10^{-5}$ \\
\hline OIV & $6.0 \times 10^{-5}$ & $3.0 \times 10^{-5}$ & $3.0 \times 10^{-5}$ \\
\hline O VI & $3.0 \times 10^{-2}$ & $1.2 \times 10^{-4}$ & $3.0 \times 10^{-5}$ \\
\hline Si IV & $6.0 \times 10^{-6}$ & $1.0 \times 10^{-5}$ & $1.5 \times 10^{-5}$ \\
\hline P IV & $1.0 \times 10^{-7}$ & $1.5 \times 10^{-7}$ & $4.0 \times 10^{-7}$ \\
\hline P V & $1.5 \times 10^{-7}$ & $1.5 \times 10^{-7}$ & $4.0 \times 10^{-7}$ \\
\hline S IV & $2.0 \times 10^{-7}$ & $3.0 \times 10^{-6}$ & $1.0 \times 10^{-5}$ \\
\hline S VI & $3.0 \times 10^{-6}$ & $3.0 \times 10^{-6}$ & $3.0 \times 10^{-6}$ \\
\hline $\mathrm{FeV}$ & $3.0 \times 10^{-5}$ & $1.0 \times 10^{-4}$ & $1.0 \times 10^{-4}$ \\
\hline $\mathrm{FeVI}$ & $2.0 \times 10^{-4}$ & $1.0 \times 10^{-4}$ & $1.0 \times 10^{-4}$ \\
\hline $\mathrm{Ni} \mathrm{V}$ & $5.0 \times 10^{-6}$ & $1.0 \times 10^{-5}$ & $1.0 \times 10^{-5}$ \\
\hline
\end{tabular}

the temperature determination from Balmer lines is difficult, as described above, and the FUSE Lyman lines favor also a somewhat higher temperature. We cannot exclude that the different abundances are due to non-LTE effects but the systematic tendency for all elements with more than one ionization stage could indicate that the temperature is indeed higher.

\section{4. $L \beta$ satellites in $C D-38^{\circ} 10980$}

Cooler DA white dwarfs show peculiar absorption features in their ultraviolet spectra, which have been identified as quasi-molecular perturbation features of $\mathrm{L} \alpha$ (Koester et al. 1985; Nelan \& Wegner 1985) or L $\beta$ (Koester et al. 1996). The features in the wing of $\mathrm{L} \beta$ near 1060 and $1078 \AA$ are caused by a perturbation of the $n=1 \rightarrow 3$ transition by protons; quantitatively the line profile can be described by the theory developed over the last 20 years by Allard and co-workers (e.g. Allard \& Kielkopf 1991; Allard \& Koester 1992; Allard et al. 1994; Koester et al. 1996; Allard et al. 1998). A comparison with observations of five DAs obtained with HUT and ORFEUS is described by Koester et al. (1998).

According to the theory, which involves the calculation of the perturbed line profiles as well as the model atmosphere calculations for DA white dwarfs, the satellite features of $\mathrm{L} \beta$ should be visible in the FUV in the temperature range of about 15000 to $25000 \mathrm{~K}$. In order to test this prediction, and also the accuracy of the predicted shape of the satellite, we have observed CD $-38^{\circ} 10980$ (WD 1620-391) with FUSE. This object is near the upper limit of the expected visibility range of the satellites. Finley et al. (1997) obtained $T_{\text {eff }}=25280 \mathrm{~K}, \log g=7.97$ from a fit to the optical Balmer lines; Vauclair et al. (1997), using a different set of optical spectra but the same model atmospheres obtained $23230 \mathrm{~K}, \log g=8.13$, and used for their analysis of HIPPARCOS parallaxes the averages of the two determinations: $T_{\text {eff }}=24250 \pm 1000 \mathrm{~K}$, $\log g=8.05 \pm 0.10$.

Ten exposures of $\mathrm{CD}-38^{\circ} 10980$ were obtained on July 13, 2000. The LiF1 channel shows fluxes that are stable from one exposure to the next, so there are probably little or no slit losses. We used the LiF1a exposures for our analysis which cover the complete region of $\mathrm{L} \beta$. Figure 4 shows the comparison of three model spectra for $T_{\text {eff }}=23000,24000,25000 \mathrm{~K}$ and $\log g=8.0$ with the observed spectrum. For the region of the higher Lyman lines, we added four of the exposures from SiC1b which show stable fluxes and are consistent with LiF1a. The model fluxed have been fitted to the observation near $1070 \AA$. 


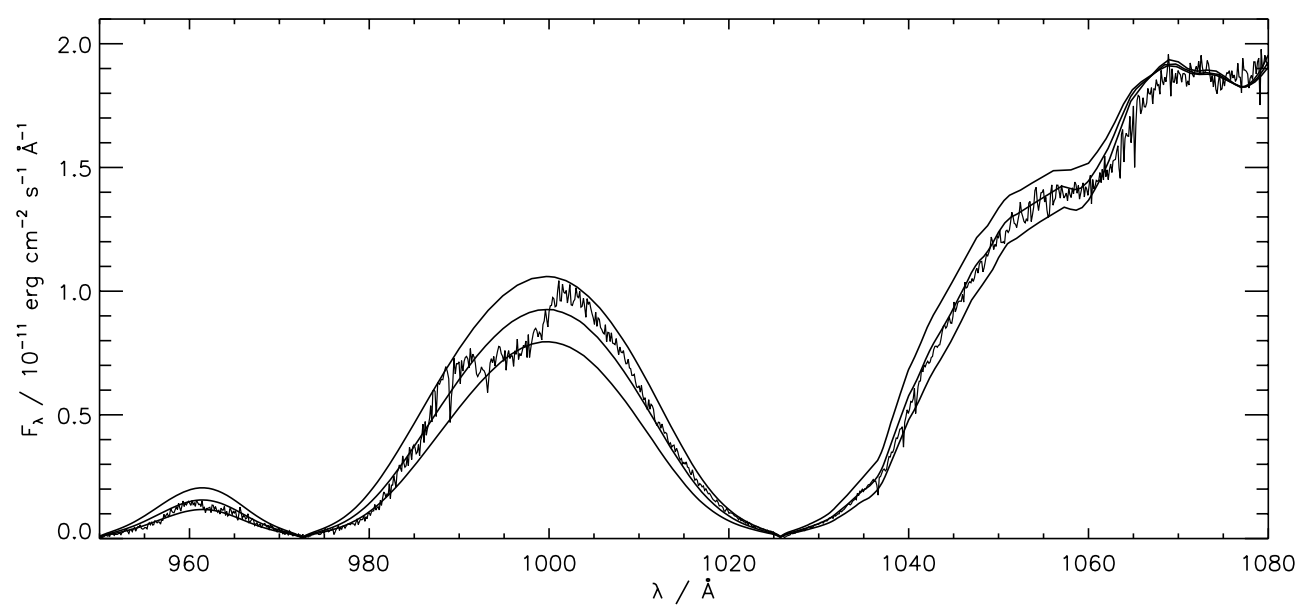

Fig. 4. FUSE spectra of CD $-38^{\circ} 10980$, rebinned to $0.15 \AA$ resolution, and compared with three model atmospheres for DA white dwarfs of $23000,24000,25000 \mathrm{~K}, \log g=8.0$, normalized at $1070 \AA$.

The $24000 \mathrm{~K}$ model gives a fairly good fit to the observed line profiles. The feature at 990 to $1000 \AA$ is a satellite feature of $\mathrm{L} \gamma$. It is visible in all channels and also in the HUT spectrum of Wolf 1346 (Koester et al. 1996) and in some ORFEUS spectra (Koester et al. 1998). It is not yet included in our models. In the 1100 to $1170 \AA$ A region, which is not shown in the figure, the fluxes are about $20 \%$ lower than the models, which we attribute to remaining absolute calibration uncertainties. The satellites are weak, but clearly visible also in this DA at about $24000 \mathrm{~K}$. This confirms the expected disappearance of the features near $25000 \mathrm{~K}$. The $1060 \AA$ satellite is very well described by the theoretical model atmosphere, which uses the line profile calculations of Allard et al. (1998) with the variable dipole moment taken into account. The $1078 \AA$ satellite unfortunately is very close to the edge of the channel and probably affected by some calibration uncertainty, but does not seem to be in conflict with theoretical prediction. The $\mathrm{SiC} 2 \mathrm{~b}$ exposure is better suited to study this feature but suffers significantly from slit losses and is, therefore, rather noisy. It seems, however, to confirm the theoretical prediction.

In addition to the Lyman series and the usual interstellar lines also three lines from Si III can be observed in the FUSE spectra: at $1108.358 \AA$, a blend at 1109.940 , $1109.970 \AA$, and a blend at $1113.204,1113.230 \AA$. The longer-wavelength member of each blend is considerably stronger, so its wavelength was used when computing the velocity. The Si III lines gave consistent results in each channel, and are at $+72 \pm 4 \mathrm{~km} \mathrm{~s}^{-1}$ with respect to the interstellar lines.

Holberg et al. (1995a) identified three different velocity systems in IUE echelle spectra of CD $-38^{\circ} 10980$ : interstellar lines with $V_{\text {ISM }}=-30.10 \pm 1.03 \mathrm{~km} \mathrm{~s}^{-1}$, circumstellar lines with $V_{\mathrm{CSM}}=+39.25 \pm 0.94 \mathrm{~km} \mathrm{~s}^{-1}$, and Si IV lines $(1393 / 1402 \AA)$ with $V_{\text {Si IV }}=+46.6 \pm 3.2 \mathrm{~km} \mathrm{~s}^{-1}$. The photospheric velocity is $V_{\text {phot }}=+51.4 \pm 2.0 \mathrm{~km} \mathrm{~s}^{-1}$. The velocity of the FUSE Si III lines is halfway between the circumstellar and photospheric components and is consistent with the Si IV lines.

The Si III lines can be reproduced using a model atmosphere of $T_{\text {eff }}=24250 \mathrm{~K}$ and $\log \mathrm{Si} / \mathrm{H}=2 \times 10^{-8}$. The same abundance can also account for the equivalent widths - as determined by Holberg et al. (1995a) - of the SiIV and the circumstellar Si III and Si II lines. The success of a single model makes a common photospheric origin of all lines plausible, although we do not have an explanation for the discrepant velocities.

\section{Discussion}

The different amounts of metals found in the FUSE spectra of the four hot DA white dwarfs correspond to the observed opacity in the extreme ultraviolet (see Wolff et al. 1998, 1999a, 1999b). The two objects with the strongest EUV absorption (MCT 0455-2812 and MCT 2331-4731) have the largest FUSE abundances whereas GD 246 shows considerably lower values. Both the EUVE and the FUSE spectra of REJ1629+78 do not exhibit signs of additional photospheric absorption. These results show that the EUV is in general a good indicator for the total amount of metals although this spectral region is mainly sensitive to the abundances of iron and nickel. A common reason for the presence of iron and other heavier elements seems therefore to be plausible. The iron abundances implied by the EUVE analysis $\left(5 \times 10^{-6}\right.$ or lower $)$ are compatible with the upper limits from the FUSE spectra.

The generally accepted explanation for heavier elements is radiative levitation. In Tables 4 and 6 , we list theoretical predictions from Chayer et al. (1995b) for the hot DAs and for Feige 55. We use the results calculated with an atmosphere contaminated by several heavier elements for the three hottest DAs. Such an atmosphere should be the best approximation to the real situation. For Feige 55, the calculations with a pure hydrogen background plasma must be taken because models with a mixed hydrogen-helium atmosphere are not available and the 
Table 6. Predicted abundances of the radiative levitation theory for Feige 55. We list the results by Chayer et al. (1995b) calculated with a pure hydrogen background plasma.

\begin{tabular}{llll}
\hline El. & $T_{\text {eff }}=60000 \mathrm{~K}$ & $T_{\text {eff }}=70000 \mathrm{~K}$ & $T_{\text {eff }}=75000 \mathrm{~K}$ \\
\hline $\mathrm{C}$ & $1 \times 10^{-5}$ & $1 \times 10^{-5}$ & $8 \times 10^{-6}$ \\
$\mathrm{~N}$ & $2 \times 10^{-5}$ & $4 \times 10^{-5}$ & $5 \times 10^{-5}$ \\
$\mathrm{O}$ & $2 \times 10^{-5}$ & $5 \times 10^{-5}$ & $6 \times 10^{-5}$ \\
$\mathrm{Si}$ & $1 \times 10^{-7}$ & $6 \times 10^{-8}$ & $5 \times 10^{-8}$ \\
$\mathrm{~S}$ & $2 \times 10^{-5}$ & $3 \times 10^{-5}$ & $3 \times 10^{-5}$ \\
$\mathrm{Fe}$ & $4 \times 10^{-5}$ & $8 \times 10^{-5}$ & $1 \times 10^{-4}$ \\
\hline
\end{tabular}

contaminated models do not extend to $T_{\text {eff }}=70000 \mathrm{~K}$ and higher.

The comparison with the observations shows the well known result that predicted and measured abundances agree rather poorly for individual elements (see e.g. Chayer et al. 1995a). The observed silicon abundances are higher than the predictions with the exception of the coolest DA RE J1629+78. A similar dependence on the effective temperature can be observed in other DAs as well (see Holberg et al. 1997 for a discussion). Sulfur has always lower measured abundances whereas the iron abundances are compatible with the predictions.

The discrepancies between observed and predicted abundances in hot white dwarf atmospheres are probably due to the limitations of previous calculations. Some of these inaccuracies could be removed by Dreizler \& Wolff (1999) who considered for the first time the influence of the absorbing ions on the atmospheric structure self-consistently. The only free parameters of their models are $T_{\text {eff }}$ and $\log g$ - the element stratification and the model spectra are determined directly by these parameters. While this model could reproduce the EUVE spectrum of G 191-B2B - which is mainly determined by iron and nickel - better than atmospheres without chemical stratification, the ultraviolet lines of carbon, nitrogen, and silicon could not be reproduced with similar accuracy. This is probably due to remaining uncertainties of the model calculations.

Another problem is the observed helium abundance in Feige 55 which is also difficult to understand with radiative levitation. The predicted abundance is lower than the observed value (Vennes et al. 1988) and a stratified hydrogen-helium atmosphere is in contradiction with the observed optical helium lines (Bergeron et al. 1994). Theoretical calculations by Unglaub \& Bues (1998) have shown that mass loss may play an important role for the presence of helium in DAO white dwarfs and the transition of DOs into DAs. Unglaub \& Bues (2000) predict for a star with the parameters of Feige $55\left(T_{\text {eff }} \approx 60000 \mathrm{~K}\right.$, $\log g \approx 7$ ) a helium abundance of $\mathrm{He} / \mathrm{H} \gtrsim 10^{-3}$. This correponds well to the measured value. If mass loss is indeed important in DAOs then it cannot be expected that the abundances of heavier elements follow the predictions of equilibrium calculations. This could explain the failure of the radiative levitation theory.

The DA white dwarfs in our sample have significantly higher gravities than Feige 55. In these objects, mass loss is probably not important. Therefore, improved equilibrium calculations are necessary to test the equilibrium radiative levitation theory.

In conclusion, we can say that the unique capabilities of FUSE complement other ultraviolet observations for studying heavy elements in hot white dwarfs. FUSE gives access to otherwise undetectable elements and provides new details in understanding the roles of radiative levitation and mass loss.

Acknowledgements. This work is based on data obtained for the Guaranteed Time Team by the NASA-CNES-CSA FUSE mission operated by the Johns Hopkins University. Financial support to U.S. participants has been provided by NASA contract NAS5-32985. BW acknowledges support by the Deutsches Zentrum für Luft- und Raumfahrt (DLR) under grant 50 OR 96173.

\section{References}

Allard, N. F., \& Kielkopf, J. F. 1991, A\&A, 242, 133

Allard, N. F., \& Koester, D. 1992, A\&A, 258, 464

Allard, N. F., Koester, D., Feautrier, N., \& Spielfiedel, A. 1994, A\&AS, 108, 417

Allard, N. F., Kielkopf, J. F., \& Feautrier, N. 1998, A\&A, 330, 782

Barstow, M. A., Fleming, T. A., Diamond, C. J., et al. 1993, MNRAS, 264, 16

Barstow, M. A., Dobbie, P. D., Holberg, J. B., Hubeny, I., \& Lanz, T. 1997a, MNRAS, 286, 58

Barstow, M. A., Holberg, J. B., Hubeny, I., \& Lanz, T. 1997b, in White Dwarfs, ed. J. Isern, M. Hernanz, \& E. GarciaBerro (Kluwer, Dordrecht), 237

Barstow, M. A., Burleigh, M. R., Bannister, N. P., Holberg, J. B., \& Hubeny, I. 2001, in 12th European Workshop on White Dwarfs, ed. H. L. Shipman, \& J. L. Provencal, ASP Conf. Ser., in press

Bergeron, P., Wesemael, F., Lamontagne, R., \& Chayer, P. 1993, ApJ, 407, L85

Bergeron, P., Wesemael, F., Beauchamp, A., et al. 1994, ApJ, 432, 305

Chayer, P., Fontaine, G., \& Wesemael, F. 1995a, ApJS, 99, 189

Chayer, P., Vennes, S., Pradhan, A. K., et al. 1995b, ApJ, 454, 429

Chayer, P., Kruk, J. W., Ake, T. B., et al. 2000, ApJ, 538, L81

Dreizler, S., \& Wolff, B. 1999, A\&A, 348, 189

Dupuis, J., Vennes, S., Bowyer, S., Pradhan, A. K., \& Thejll, P. 1995, ApJ, 455, 574

Finley, D. S., Koester, D., \& Basri, G. 1997, ApJ, 488, 375

Holberg, J. B., Bruhweiler, F. C., \& Andersen, J. 1995a, ApJ, 443, 753

Holberg, J. B., Saffer, R. A., Tweedy, R. W., \& Barstow, M. A. 1995b, ApJ, 452, L133

Holberg, J. B., Barstow, M. A., Lanz, T., \& Hubeny, I. 1997, ApJ, 484, 871

Holberg, J. B., Barstow, M. A., \& Sion, E. M. 1998, ApJS, 119, 207 
Koester, D., Weidemann, V., Zeidler-K. T., E.-M., \& Vauclair, G. 1985, A\&A, 142, L5

Koester, D., Finley, D. S., Allard, N. F., Kruk, J. W., \& Kimble, R. A. 1996, ApJ, 463, L93

Koester, D., Sperhake, U., Allard, N. F., Finley, D. S., \& Jordan, S. 1998, A\&A, 336, 276

Lamontagne, R., Wesemael, F., Bergeron, P., et al. 1993, in White Dwarfs: Advances in Observation and Theory, ed. M. A. Barstow (Kluwer, Dordrecht), 347

Lanz, T., Barstow, M. A., Hubeny, I., \& Holberg, J. B. 1996, ApJ, 473, 1089

Moos, H. W., Cash, W. C., Cowie, L. L., et al. 2000, ApJ, 538, L1

Nelan, E. P., \& Wegner, G. 1985, ApJ, 289, L31

Sahnow, D. J., Moos, H. W., Ake, T. B., et al. 2000, ApJ, 538, L7
Sion, E. M., Holberg, J. B., Barstow, M. A., \& Kidder, K. M. 1995, PASP, 107, 232

Unglaub, K., \& Bues, I. 1998, A\&A, 338, 75

Unglaub, K., \& Bues, I. 2000, A\&A, 359, 1042

Vauclair, G., Schmidt, H., Koester, D., \& Allard, N. 1997, A\&A, 325, 1055

Vennes, S., Pelletier, C., Fontaine, G., \& Wesemael, F. 1988, ApJ, 331, 876

Vennes, S., Chayer, P., Hurwitz, M., \& Bowyer, S. 1996, ApJ, 468,898

Wolff, B., Koester, D., Dreizler, S., \& Haas, S. 1998, A\&A, 329, 1045

Wolff, B., Koester, D., \& Dreizler, S. 1999a, in 11th European Workshop on White Dwarfs, ed. J.-E. Solheim, \& E. G. Meištas, ASP Conf. Ser., 169, 524

Wolff, B., Koester, D., \& Lallement, R. 1999b, A\&A, 346, 969 